\title{
ANALYSIS OF TECHNICAL CONDITION ASSESSMENT OF GAS TURBINE BLADES WITH NON-DESTRUCTIVE METHODS
}

\author{
Józef BŁACHNIO* \\ *Bialystok University of Technology, Faculty of Mechanical Engineering, ul. Wiejska 45C, 15-351 Bialystok, Poland
}

j.blachnio@pb.edu.pl

\begin{abstract}
Structural components of gas turbines, particularly the blades, sustain a variety of damages during the operation process. The most frequent cause of these damages are the overheating and thermal fatigue of the material. A primary technique to assess condition of the blades is the metallographic examination. In spite of the fact that metallographic analysis delivers much more information on the structure of examined blade material, it is a type of destructive test resulting in the destruction of the blade which makes further utilization of the item impossible. The paper has been intended to discuss non-destructive testing methods and to present capabilities of applying them to diagnose objectively changes in the microstructure of a turbine blade with computer software engaged to assist with the analyses. The following techniques are discussed: a visual method, based on the processing of images of the material surface in visible light, active thermography, based on the detection of infrared radiation, and the X-ray computed tomography. All these are new non-destructive methods of assessing technical condition of structural components of machines. They have been intensively developed at research centers worldwide, and in Poland. The computer-aided visual method of analyzing images enables diagnosis of the condition of turbine blades, without the necessity of dismantling of the turbine. On the other hand, the active thermography and the X-ray computed tomography, although more sensitive and more reliable, can both be used with the blades dismounted from the turbine. If applied in a complex way, the non-destructive methods presented in this paper, are expected to increase significantly probability of detecting changes in the blade's condition, which in turn would be advantageous to reliability and safety of gas turbine service.
\end{abstract}

Key words: Gas Turbine, Blade, Diagnosing, Technical Condition

\section{INTRODUCTION}

A turbine is a rotary flow machine, converting enthalpy of the working medium, also called the working fluid (exhaust stream, gaseous decomposition products or compressed gas) into the mechanical work, causing rotation of the rotor. Because of numerous advantages, such as the ability to develop high power in a compact and low curb weight, relatively high efficiency of energy conversion of the process (30-45\%), simple structure, ease of use in different climatic conditions (particularly at low temperatures of the surrounding medium), and a fairly large reliability, the turbines found many applications, among others in power industry, traction motors, marine and aviation engines as well as in aerospace technology. Unfortunately, gas turbines also have drawbacks, which mainly include high operating temperature of some of their parts such as blades and large rotor speeds. Currently, depending on the materials of which the blades are made and treatments such as cooling or blade coating using special heat resistance coating (Skočovský et al., 2004), the temperature of the gas turbine working medium ranges from 1100 to $1600 \mathrm{~K}$, and in some designs it is even higher (Pike and Flower, 2006). Moreover, the rotational speed of, for instance, aerial turbine engines, depending on their design and purpose can reach as much as $100 \mathrm{k}$. rpm or even more.

In the process of operation, different types of damage to the gas turbine components may occur (Błachnio and Kułaszka, 2009; Błachnio, 2011; Karczewski, 2008; Szczepanik and Rządkowski, 2012). The parts of the turbine that are the most vulnerable to damage are blades, whose technical condition is crucial for the reliability and service life of the entire turbine and the assembly, in which it is embedded. The main causes of a gas turbine's damages are its overheating and thermal fatigue (Fig. 1). a)

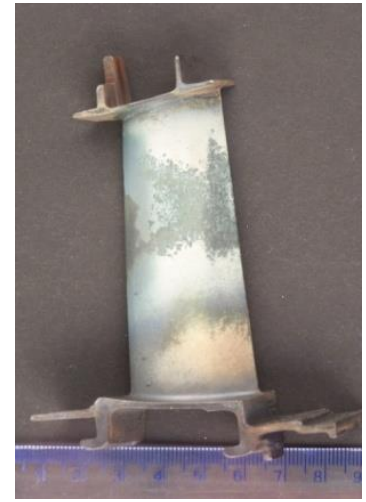

b)

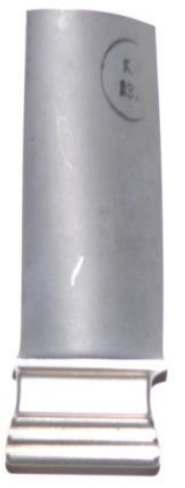

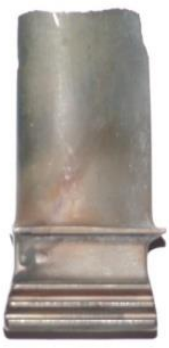

Fig. 1. a) - a vane with overheated area, b) - an image of the blade clipped due to overheating (Błachnio and Kułaszka, 2009; Błachnio, 2011)

This is mainly due to adverse operating conditions or manufacturing defects, such as use of insufficiently durable types of protective coating or applying them on the blade material in a wrong way (Błachnio and Pawlak, 2011; Burakowski, 2002; Maldague, 2001). The reaction of the blade material to mechanical loads depends mainly on its operating temperature. The choice of material for manufacturing blades with a projected durability must include their mechanical properties in the area of the 
maximum temperature on a given blade. A typical temperature distribution along the blade feather is very uneven (Fig. 2). Damages to the first (central) stages of a turbine are usually caused by the impact of high temperature of exhaust, whereas damages to the last, (peripheral) stages ( turbines with the longest blades) originate mainly from the effects of mechanical strain (vibration, centrifugal force) (Błachnio, 2011; Karczewski, 2008; Szczepanik and Rządkowski, 2012; Marsh, 2013).

Overheating of the blades is the result of the exceeding of the permissible average exhaust gas temperature value as well as non-uniform temperature distribution at the periphery. One of the reasons for the irregularity of the temperature behind the turbine is not appropriate fuel spray caused by varnish deposits accumulating on the injectors (Błachnio and Pawlak 2011).

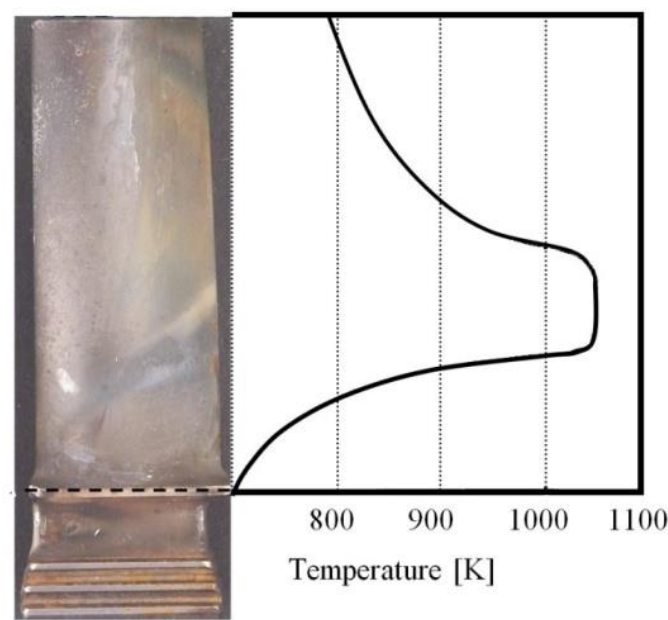

Fig. 2. A diagram of the typical temperature distribution along the gas turbine blade feather (Błachnio, 2011)

An occurrence of the failure of the gas turbine leads to its destruction. This situation forced the constructors and researchers of the turbine engines to develop non-destructive methods of evaluating the turbine's condition (Maldague, 2001). So far, the most common method is a visual one (Błachnio and Kułaszka, 2009; Karczewski, 2008). It allows for testing of overheating structures even of those hard-to-reach engine components in a noninvasive way, however, with relatively small reliability (Pitkanen et al., (2001)). The assessment of the state of overheated blade is performed visually by a diagnostician using videoscope, and comparing the resulting image with the reference one. This type of diagnosis, however, is fraught with subjective error, therefore, the diagnostician's decision is verified by performing a destructive metallographic examination.

An important task of the aircraft engines use is to adopt maximally available diagnostic methods and knowledge in order to detect early enough and interpret the symptoms of the possible risks, to perform repairs in the most favourable moment in respect of minimizing losses, as well as to prevent failures (Spychała et al., 2013; Żółtowski and Cempel, 2004). Furthermore, the high cost of repair of aircraft engines has become one of the reasons for developing new non-destructive assessing methods performed in the process of supported operating as well as during the verifications, when repairing turbine components is being conducted.

\section{VISUAL METHOD BASED ON PROCESSING IMAGES OF THE SURFACE OF THE TURBINE BLADES IN VISIBLE LIGHT}

Visual method is commonly used in a non-destructive examination of technical objects (Błachnio and Kułaszka, 2009; Karczewski, 2008). In the case of the use of optical instruments, and in particular a videoscope, it is possible to inspect the object without necessity of disassembling it. The acquisition and development of information that could be useful for the diagnosis of machine parts, is a complex process that requires multiple treatments. In general this process can be summarized as follows (Błachnio and Bogdan, 2010; Bogdan et al., 2009):

- Illumination of the diagnosed object located in the background with white light,

- Acquisition of the test object image,

- Digital analysis of the image,

- Presentation of information on the image.

Illuminated surface of the blade can be recognized by a lightsensitive detector (CCD matrix with an optical system - optoelectronic system) through a secondary source of light from its surface. CCD matrix counts incident photons, energy of the light rays incident on each pixel. In the case of a computer vision system the image is obtained from ambient by means of an optoelectronic device - videoscope (Fig. 3). This enables indirect method of diagnosis of the object through the processing and analysis of data collected in the form of digital images.

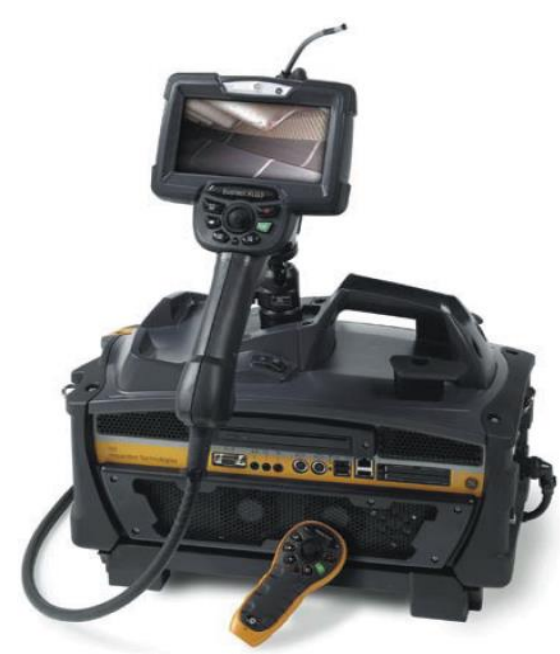

Fig. 3. Optoelectronic device - videoscope (EVEREST XLG3-Video Probe. 2006)

Features of digital images allow to determine their histogram, i.e. the frequency chart of successive values of pixels in the image. The histogram shows how numerous are points in the image with different brightness values lk (Adamczyk and Będkowski 2005; Bogdan et al., 2009). It is assumed that the first element of the histogram has the number 0 , and the last is equal to the number of levels of image brightness. If the image contains $m \times n$ points and is saved using $P$ levels of gray (for monochrome image):

$p_{m}=\left\{0,1,21, \quad, l_{k}, \quad, 254,255\right\}$ and for the RGB color image:

$p_{R}=\left\{0,1,21, \quad, l_{k}, \quad, 254,255\right\}$,

$p_{G}=\left\{0,1,21, \quad, l_{k}, \quad, 254,255\right\}$,

$p_{B}=\left\{0,1,21, \quad, l_{k}, \quad, 254,255\right\}$, then the usual result is $m \times n / p$ that 
have a certain level of gray or shades of colour component in the case of colour images. The histogram is represented as a vector of length corresponding to the number of brightness levels, it can be written as follows (Wróbel and Koprowski, 2004):

$$
h\left(l_{k}\right)=\sum_{m=1}^{M} \sum_{n=1}^{N} p\left(l_{k},(m, n)\right)
$$

where: $h\left(l_{k}\right)$ - the sum of points, that level of gray equals $l_{k}$ and

$$
\mathrm{p}\left(\mathrm{l}_{\mathrm{k}},(\mathrm{m}, \mathrm{n})\right)=\left\{\begin{array}{l}
1 ; \text { for } \mathrm{L} 1(\mathrm{~m}, \mathrm{n}) \neq \mathrm{l}_{\mathrm{k}} \\
0 ; \text { for } \mathrm{L} 1(\mathrm{~m}, \mathrm{n}) \neq \mathrm{l}_{\mathrm{k}}
\end{array}\right.
$$

Disruptions that occur in all stages of the process of acquiring and analyzing information impinge on the reduction of the efficiency of data acquisition in the form of digital images. The following assumptions for research are being assumed:

- Acquisition of images in the same conditions and the same resolution,

- Illumination - diffuse white light,

- No light interference - reflected from other surfaces,

- Acquisition of images using a videoscope of the same resolution at the same magnification.

Representative areas (averaged ROI areas - regions of interest) are selected to describe the tested surfaces unequivocally. By means of the Matlab software (Image Processing Toolbox) image of the blade surface is transformed into the RGB colour image or grayscale image. Typically, the test of image histogram is carried out in order to determine whether the "black-white" information is sufficient to describe the change of the colour caused by the action of high temperature (superheat).

Parametric description of histograms is to determine the position of the maximum value of the amplitude, i.e. the number of pixels (ordinate axis) for the values of saturation of grayscale or RGB (abscissa axis) - Fig. 4. In order to extract diagnostic information the examined waveforms of the changes of the maximum amplitude values in the function of temperature are approximated by polynomials.

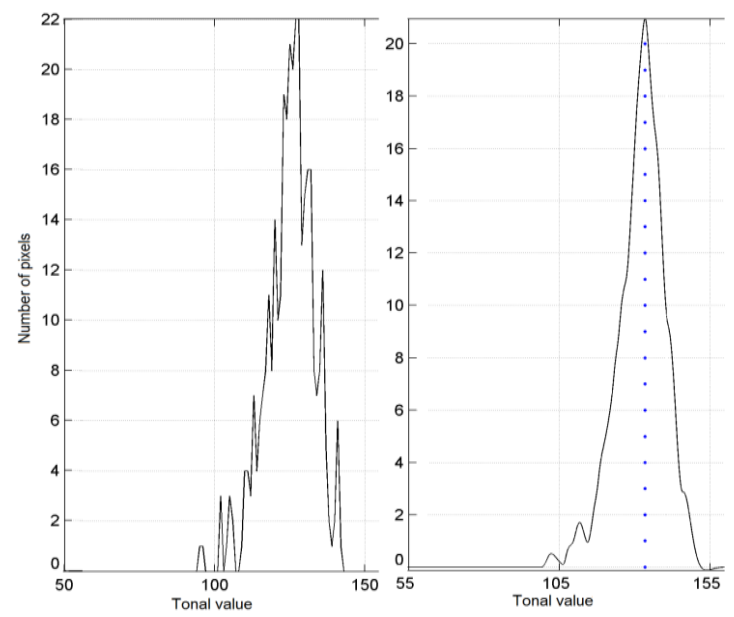

Fig. 4. Exemplary spectral analysis of the image of blade surface (Błachnio and Bogdan, 2010)

Acquisition by videoscope and computer analysis of images of the surface samples of the blades made of superalloy El-867 WD were performed before and after heating in a furnace in realistic conditions. Studies of the first order statistics (parametric description of histograms) of images of blades surfaces showed a monotonic decrease of values of the amplitude of grayscale saturation (Fig. 5).

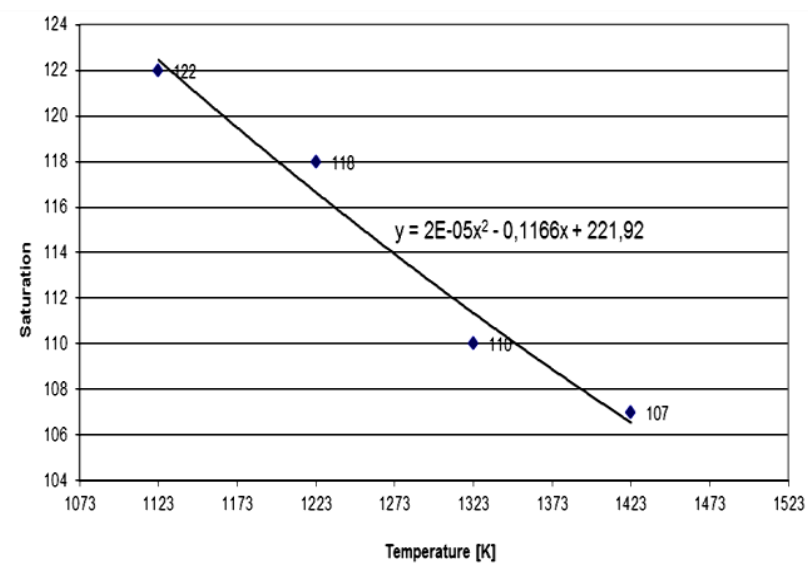

Fig. 5. The values of the position of the maximum amplitude of greyscale saturation of blade surfaces annealed in conditions similar to realistic (Błachnio and Bogdan, 2010)

\section{ACTIVE THERMOGRAPHY}

Active thermography is based on the detection of infrared radiation. The essence of the study is to analyze the thermal response of the material to external impulse heat stimulation. If a certain amount of energy is delivered to the surface of the material, for example in the form of a heat pulse, after its expiry the surface temperature will start changing rapidly. Due to thermal diffusion, thermal front will move into the material. The presence of areas with different thermal properties (including defects) compared with areas without defects causes a change in the diffusion rate. Thus, by monitoring the temperature field on the surface of the cooling sample, it is possible to reveal position of the defects. Depending on the stimulation method, there are a few types of active thermography, namely, pulsed thermography, lock-in thermography with modulated heating and pulsed phase thermography (Oliferuk, 2008; Holland, 2011). Pulsed thermography is considered to be a relatively simple kind of active thermography. It depends on the determination and analysis of the temperature distribution in the study area during its cooling down after prior uniform heating with the use of thermal pulse (Fig. 6).

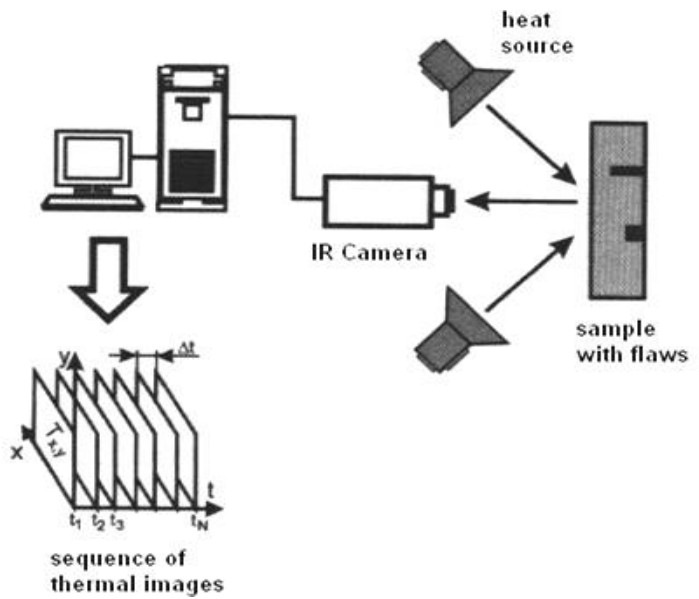

Fig. 6. Application diagram for the pulsed thermography (Oliferuk, 2008) 
For one-dimensional model and homogeneous material, the expression of the temperature change during the cooling of the surface heated by short thermal pulse, is as follows (Oliferuk, 2008):

$T(t)-T(0) \sim Q \alpha^{-0.5} t^{-0.5}$

where $Q$ is the heat pulse energy per unit of area, $t$ - surface cooling time, $T(0)$ is the temperature at a selected point or area of the heated surface, just after extinguishing the pulse, and $T(t)$ - the temperature in the cooling process at any time.

The occurrence of defects in the material reduces the diffusion rate which means that temperature of the surface area above the defect will be different from the temperature of the region under which there are no defects - and therefore, the aspect of the above relationship changes. This method (as well as others), has limitations, since due to rapidly fading with depth the temperature contrast it only allows for the detection of subsurface flaws.

Thermographic examinations covered the turbine blades made of alloy EL-867WD, classified into various categories: new, used - fit and damaged during turbine engine operation. The obtained results confirmed changes of parameter dependence of thermal response of the examined blade materials to stimulating heat pulse (Fig. 7).
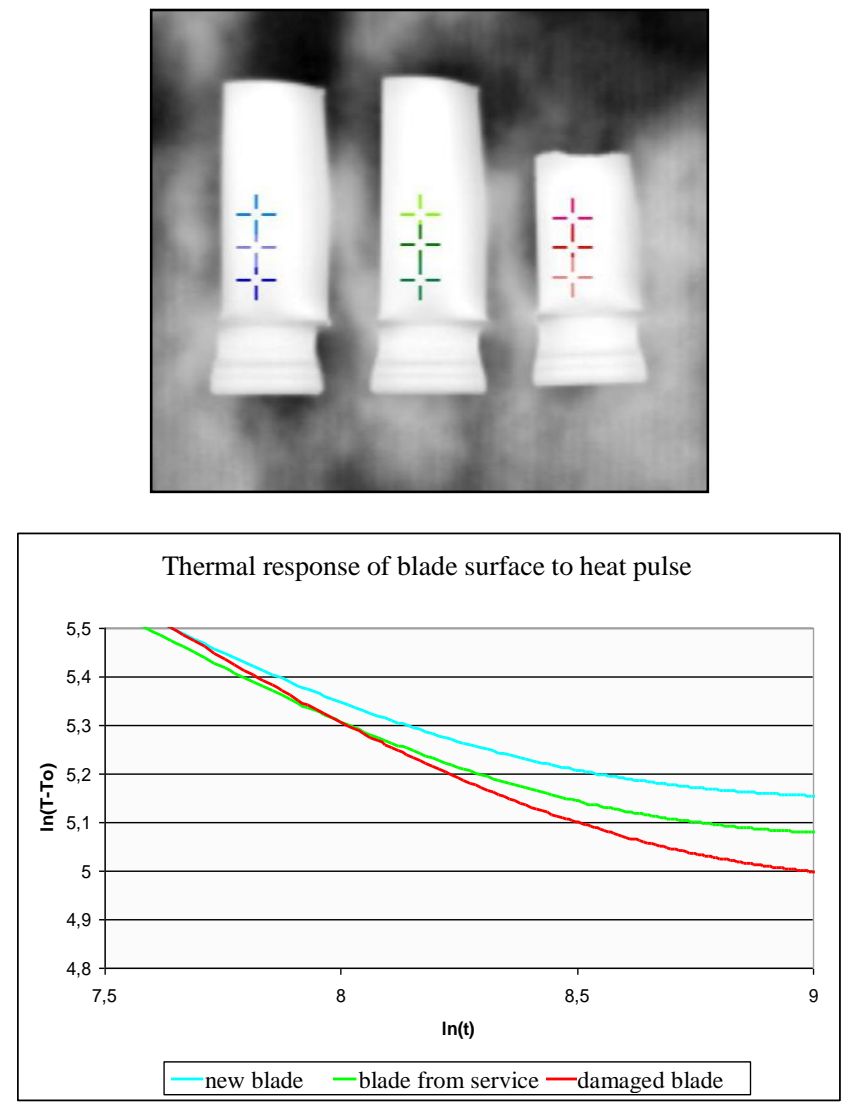

Fig. 7. Blades thermograph and changes of dependence of the parameter $\ln (\mathrm{T}-\mathrm{To})$ of the thermographic signal at selected points of the blade surfaces (Kułaszka et al., 2011)

While on the basis of the analysis of the response to the heat pulse of the blade material classified as "unfit", it was possible to determine areas clearly deviating from the average value. These areas coincided with areas visually assessed as overheated which, combined with the results of the tests conducted on sam- ples (Fig. 7) allows to draw conclusions on superheated structure of the material.

\section{X-RAY COMPUTER TOMOGRAPHY}

Tomography is the collective name for the diagnostic methods aimed at obtaining a spatial image of the machine element. In the technical diagnosis, a method of computed tomography (called CT) has been widely applied (The research report, 2011). It is a kind of X-ray tomography allowing for obtaining spatial images (3D) from the radiograph scanning of the object performed from different directions (Fig. 8).

By using the tomograph and a computer implemented program, an object tomogram is obtained. CT is now widely used in industrial production quality control process as a non-destructive method. In the computer tomography radiation source is X-ray tube. The X-ray detectors used in computer tomography are mainly ionization chambers and scintillators. In the scintillation detector, ionizing radiation creates excitation of atoms or scintillator molecules which in turn, by releasing the energy obtained this way, emit quanta of the radiation in the visible range. The light signal must then be converted into the electric signal by a photomultipliers or photodiodes. By using the X-ray source there are performed a series of radiographies with a beam of X-rays. The data stream from the detectors contains information about the absorption (scattering) of radiation through the various components of the test object. These data are stored in computer memory and digitally analyzed in order to obtain monochrome images.

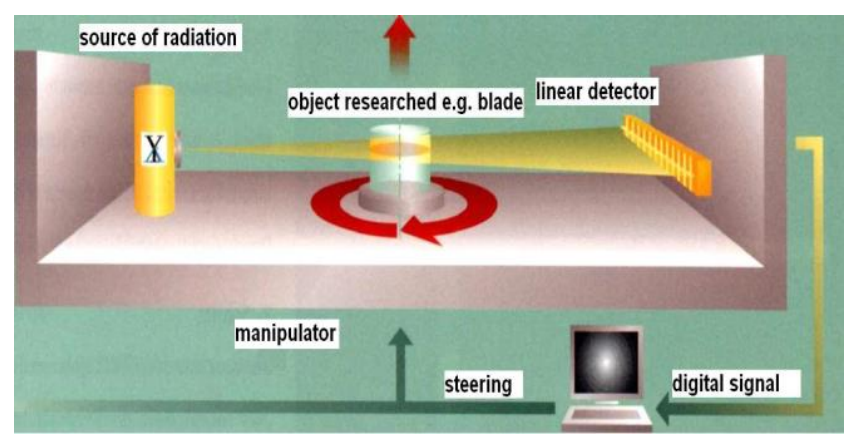

Fig. 8. The example of a tomography investigation with use of a linear detector (Electronic Instrument CT-X-ray, 2011)

Each cross section of X-rays through object of study is divided into small parts - voxels. For each voxel there is assigned a numerical value proportional to the degree to which it absorbs radiation. To determine this value for $n$ voxels there is a need of at least $\mathrm{n}$ of equations describing the absorption in a given layer. $\mathrm{N}$ different cross-layer projections are needed. The more projections, the better image accuracy is achieved. The most commonly used image reproducing methods are analytical methods. They are able to yield the best results, but require large computing power. Two-dimensional Fourier analysis method uses fast Fourier transform to describe obtained absorption profiles. Transformation is subjected to each of the projection, and thus the absorption coefficient in each of the voxels is obtained. The absorption coefficients are converted to CT numbers, also called HU Hounsfield units (The research report, 2011): 


$$
1 \mathrm{HU}=K \frac{\mu_{p}-\mu_{w}}{\mu_{w}}
$$

where: $K$ - constant image enhancement (individual for each tomography) $\mu_{p}$ - pixel absorption coefficient $\mu_{w}$ - the coefficient of water absorption (reference value).

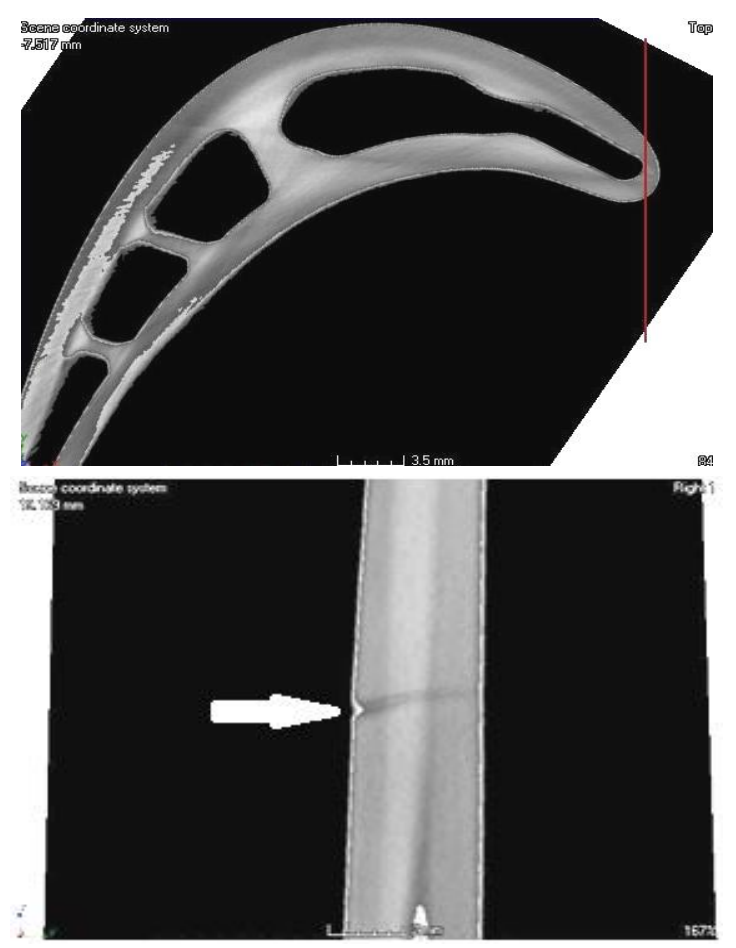

Fig. 9. Tomogram of a turbine blade with a visible crack at the leading edge (Chalimoniuk et al., 2013)

In order to obtain high accuracy of projection the internal structure of machine element, the radiation beam is limited by the diaphragm to a flat beam and a digital linear detector is used (one series of sensors). After rotating the element by $360^{\circ}$ a flat X-ray image of slice is obtained. In order to obtain a spatial image of the entire element, the element is shifted in a vertical plane, and at each step a full turn is made. A 3D image is obtained after processing of all collected data.

Computer radiography applied to turbine blades allows the observation of actual thickness of the inner, invisible walls (Chalimoniuk et al., 2010). An image of the object can be represented in the colours and the shape of the inner wall can be determined in the appropriate scale, e.g., the size and location of the defect. Also, the geometry of the internal components can be accurately measured and the tolerance of their actual dimensions be assessed. Thus, CT scan allows to verify the correctness of realization of the component with a very high degree of accuracy and diagnose internal damages, for example, cracks, blade canals blockage, etc. (Fig. 9).

\section{SUMMARY}

Various types of damage to the components of gas turbines occur in the process of their operation. Of all parts of the turbine, the most vulnerable to damage are blades, whose condition has a significant impact on the reliability and durability of the entire engine and assembly, in which it is embedded. The main causes of damage to the blades of the gas turbine are their overheating and thermal fatigue. The destruction of the gas turbine blades usually starts with the destruction of the protective coating and, as a result, the blade base material is exposed to direct aggressive impact of exhaust gases. This situation leads directly to overheating of the material, seen as unfavorable changes in the microstructure. As a result, the surface roughness changes, and in particular the colour of the blade surface. The color of the surface is therefore a diagnostic signal, which is used in the visual method. Using computer analysis of image recognition, one can objectively evaluate change in the blade state, such as overheating based on the analysis of the colour changes in its surface. Visual method thus enables diagnosis of the status of the blades without necessity to disassemble the turbine.

Results of the study on gas turbine blades, the new and the used ones, after using an active thermography showed the existence of dependencies and relationships between operational heat load and signals changes of the material thermal response. These dependencies and relationships are used to assess the state of overheating of the gas turbine blade material. Although this method is more sensitive and reliable, however, it can be used after disassembling the blades from the turbine.

Computer tomography allows achieving spatial images of the test objects state in a relatively short period of time. It is characterized by high credibility and reliability. Like the previous method it can be used after disassembling the blades from the turbine. In addition, costly X-ray CT scanner is required for the research.

The use of presented non-invasive methods, especially in a complex manner, significantly increase the probability of detecting condition changes of the blades, which will increase the reliability and safety of operation of gas turbines.

\section{REFERENCES}

1. Adamczyk J., Będkowski K. (2005), Digital methods in remote sensing, SGGW Publishing House, Ed. 1, Warsaw.

2. Błachnio J. (2011), Analysis of causes of decohesion of a gas turbine blade made of El-867WD alloy, Aircraft Engineering and Aerospace Technology, An International Journal, Vol. 83 No 1, 14-20.

3. Błachnio J., Bogdan M. (2010), A non-destructive method to assess condition of gas turbine blades, based on the analysis of blade-surface image, Russian Journal of Nondestructive Testing, Vol. 46, No. 11, 860-866.

4. Błachnio J., Kułaszka A. (2009), Computer aided visual inspection of the technical condition of gas turbine blades during their operation period, Journal of KONES, Vol. 16, No. 3, 23-30.

5. Błachnio J., Pawlak W. (2011), Damageability of gas turbine blades - evaluation of exhaust gas temperature in front of the turbine using a non-linear observer, Advances in Gas Turbine Technology, In Tech, 435-464.

6. Bogdan M., Błachnio J., Derlatka M. (2009), Computer-aided method of diagnostics of gas turbine blades, Acta Mechanica et Automatica, Vol 3, No. 4, 13-16.

7. Burakowski T. (2002), The proposal of determining quantitative synergism in surface engineering, $3^{\text {rd }}$ International Conference on Surface Engineering, Chengdu, P. R. China.

8. Chalimoniuk M., Błachnio J., Krzysztofik J. (2010), Analysis of the feasibility to investigate condition of gas turbine vanes by means of the radiographic method, Journal of KONBIN, No 1(13), 129-138.

9. Chalimoniuk M., Szczepanik R., Błachnio J. (2013), The rate of decohesion of a gas turbine blade as assessed with the X-ray computed tomography (CT), Journal of KONES, Vol. 20, No. 3, 89-96. 
10. Electronic Instrument CT-X-ray (2011), The research report, Computer Tomography for Industrial Applications, YXLON. International.

11. EVEREST XLG3-Video Probe (2006), GE Inspection Technologies.

12. Holland S. D. (2011), Thermographic signal reconstruction for vibrothermography, Infrared Physics \& Technology, 54, 503-511.

13. Karczewski, Z. (2008), Endoscopic diagnostics of marine engines, Diagnostyka, 3(47), 19-23.

14. Kułaszka A., Bogdan M., Błachnio J. (2011), New non-destructive methods of diagnosing health of gas turbine blades, Advances in Gas Turbine Technology, InTech, 465-498.

15. Maldague X. (2001), Theory and practice of infrared technology for nondestructive testing, John Wiley and Sons, New York.

16. Marsh S. (2013), Preventig fretting fatigue in blade dovetail roots by modifying geometry of contact surfaces, Power Transmission Engineering, 45-49.

17. Oliferuk W. (2008), Infrared thermography for nondestructive testing of materials and equipment, Gamma Office, Warsaw.

18. Pike L. M., Flower H. L. (2006), Gas turbine superalloy with improved fabricability. Advanced Materials and Processes, ASM International, Vol. 164, No. 6, 39- 43.

19. Pitkänen J. et al. (2001), NDT methods for revealing anomalies and defects in gas turbine blades, Proc $15^{\text {th }}$ WCNDT, Rome.
20. Skočovský P., Podrábský T., Belan J. (2004), Operational degradation of aluminium-silicone layer of turbine blades made from Ni-based alloy, The Archive of Machinery Technology and Automation, Vol. 24, No. 1, 45-52.

21. Spychała J., Pawlak W., Kułaszka A., Błachnio J. (2013), Assessment of technical condition demonstrated by gas turbine blades by processing of images for their surfaces, Journal of KONBIN, $1(21)$.

22. Szczepanik R., Rządkowski R. (2012), A study on the dynamics of aero engine blades under different operating conditions, Air Force Institute of Technology, Warsaw.

23. Wróbel Z., Koprowski R. (2004), Practice in image processing within the MATLAB software, EXIT Academic Publishing House, Warsaw.

24. Zieliński T. P. (2005), Digital signal processing - from theory to practice, Transport and Communications Publishers, Warsaw.

25. Żóltowski B., Cempel C. (2004), Machinery fault diagnosis engineering, Polish Society of Engineering Diagnosis, Warsaw. 